\title{
RÉFLEXIONS ET PRATIQUES RELATIVES À LA VARIATION TOPOLECTALE EN TERMINOLOGIE
}

\author{
Annie Garneau \\ Robert Vézina ${ }^{1}$
}

\begin{abstract}
RESUMO: A variação lexical, seja temporal, social ou geográfica, preocupa cada vez mais os terminólogos e lexicógrafos interessados pelas línguas consideradas de grande difusão. Sabemos que o registro dialetal permite identificar, tanto nos dicionários de língua geral quanto nas obras de caráter terminológico, o uso particular de uma unidade lexical segundo diferentes eixos de variação. Esse artigo trata da variação lexical geográfica ou variação topolectal e, de maneira mais precisa, das práticas terminológicas na língua francesa. Por meio de um breve resumo histórico sobre o uso desse fenômeno, apresentamos os diferentes tipos de registros topolectais utilizados nas obras terminológicas francófonas e multilíngües. O exame desse contexto específico nos conduz também à formulação de algumas observações sobre a maneira de apresentar os termos de línguas diferentes numa obra multilingüe.
\end{abstract}

UNITERMOS: Terminologia francófona; variação topolectal; registro topolectal; variação lexical geográfica; terminologia multilingüe.

Annie Galarneau et Robert Vézina sont tous deux terminologues à l'Office québécois de la langue française, Québec (Canada). 
RÉSUMÉ: La variation lexicale, qu'elle soit temporelle, sociale ou géographique, occupe de plus en plus de place dans les préoccupations des terminologues et lexicographes intéressés par les langues de grande diffusion. On sait que le marquage linguistique permet d'identifier, dans les dictionnaires de langue générale comme dans les ouvrages à caractère terminologique, l'usage particulier qui est fait d'une unité lexicale selon différents axes de variation. C'est précisément à la variation lexicale géographique, ou topolectale, et en particulier au traitement qui en est fait en terminologie de langue française que s'intéresse cet article. À travers un bref historique de l'évolution de la prise en compte du phénomène, nous faisons état des différents types de marquage topolectal utilisés dans les travaux terminologiques francophones et multilingues. L'examen de ce contexte particulier nous conduit également à formuler quelques observations relatives à la façon de présenter les termes de langues différentes dans un ouvrage multilingue.

MOTS-CLÉS: Terminologie francophone; variation topolectale; marquage topolectal; variation lexicale géographique; terminologie multilingue.

\section{Introduction}

La prise en compte de la variation lexicale dans le travail terminologique et lexicographique est devenue au fil des années une des préoccupations importantes des terminologues et des lexicographes s'intéressant aux langues de grande diffusion. Ainsi, dans un dictionnaire de langue générale ou à caractère terminologique, on a généralement recours au marquage linguistique pour décrire plus précisément l'usage qui est fait d'une unité lexicale, selon qu'on l'analyse d'un point de vue temporel, social ou géographique.

TradTerm, 14, 2008, p. 193-220 
C'est d'abord de la variation lexicale géographique, ou topolectale, qu'il sera question dans cet article, et plus précisément du traitement qui en est fait en terminologie de langue française. La variation lexicale géographique peut être rapidement définie comme étant l'ensemble des différences qui touchent le vocabulaire d'une langue (sa composition ou son utilisation) en fonction des territoires où elle est en usage (continents, pays, régions, États, localités, etc.). En général, plus une langue est parlée sur un vaste territoire, plus cette variation géographique est perceptible.

De manière à situer l'état actuel des réflexions sur ce type de variation en français et sur la pratique terminologique qui en découle, nous tracerons un bref portrait historique de l'évolution de la prise en compte du phénomène, principalement à travers l'expérience québécoise. Nous serons ainsi amenés à faire état des différents types de marquage topolectal qui sont utilisés dans la réalisation de travaux terminologiques francophones. Nous poursuivrons en abordant la problématique du marquage topolectal dans un contexte de terminologie multilingue. L'examen de ce contexte particulier nous conduira également à formuler quelques observations relatives à la façon de présenter les termes de langues différentes dans un ouvrage multilingue.

\section{I- La prise en compte de la variation topolectale}

\section{1- Un rappel historique}

La prise en compte de la variation topolectale en terminologie et en lexicographie de langue française a connu une évolution notable au fil des ans. De façon générale, l'histoire de la lexicographie française montre que les auteurs de dictionnaires ont eu tendance à proposer une version uniforme et monolithique de la langue et à négliger les particularismes propres au français parlé en dehors de Paris. Mis à part Furetière et 
Guérin ${ }^{2}$ qui ont intégré, dans leurs dictionnaires, des particularismes propres aux variétés de français parlées hors de France, les lexicographes français se sont montrés plutôt réticents à cette pratique, et ce jusqu'à la fin des années 70 . Conformément à cette vision plutôt centralisatrice de la langue, le français du Québec, comme d'ailleurs le français de Belgique et le français de Suisse, a été généralement perçu et décrit comme une variété régionale du français ${ }^{3}$.

Dans les années 60, l'Office de la langue française ${ }^{4}$ (créé en 1961) partage essentiellement cette vision des choses. Toutefois, il considère que l'usage doit s'aligner sur le français international (qui, dans les faits, correspond essentiellement au français de Paris), tout en faisant sa place à l'expression des réalités spécifiquement nord-américaines (Office de la langue française, 1965:6). L'ouverture à l'expression des réalités nordaméricaines est cependant fortement encadrée, et les canadianismes ne sont acceptés que dans la mesure où ils sont sentis comme utiles et pratiquement irremplaçables (Vézina, 2005:272).

Le même principe est appliqué dans les travaux terminologiques effectués au Québec; ceux-ci sont d'abord conduits dans une optique d'alignement sur le français en usage en France, orientation pondérée toutefois d'une certaine tendance à l'intégration des canadianismes. C'est ainsi que Fernand Sylvain et ses collaboratrices (1977), en publiant le Dictionnaire de la comptabilité, tentent de normaliser la terminologie comptable en usage au Canada; en proposant une terminologie utilisée ailleurs dans la francophonie, ils font une place à quelques termes propres à la terminologie comptable employée au Canada.

2 En effet, Antoine Furetière, dans son Dictionnaire universel (1727), tient compte de la réalité canadienne et notamment du vocabulaire en usage dans les relations de voyages de l'époque et, deux siècles plus tard, Paul Guérin introduit dans son Dictionnaire des dictionnaires (18841890), et particulièrement dans son Supplément (1895), un nombre considérable de mots et d'emplois canadiens.

3 Voir à ce sujet Galarneau (2000).

4 Depuis 2002, l'Office de la langue française porte le nom d'Office québécois de la langue française.

TRadTerm, 14, 2008, p. 193-220 
À partir des années 80 surtout, on constate une volonté accrue de valorisation des différentes variétés de français hors de France; du coup, la pratique terminologique et lexicographique francophone tient compte de façon plus importante du phénomène de la variation géographique. Cette plus grande ouverture face aux différences d'ordre lexical qui marque le paysage francophone est en partie causée par les contacts de plus en plus étroits entre les différentes communautés ayant le français en partage, ainsi que par une reconnaissance de l'importance des identités culturelles. Le français du Québec, le français de Belgique et le français de Suisse, notamment, sont désormais définis comme des variétés nationales de français, et les désignations de leurs particularités lexicales sont appelées respectivement québécismes, belgicismes et helvétismes (Hausmann, 1986:4-5). En ce qui touche la production terminologique effectuée à l'Office de la langue française, on constate que les québécismes tendent déjà à être évalués en fonction non seulement du respect de la grammaire française et du vocabulaire français commun, mais aussi en fonction des pratiques linguistiques des locuteurs québécois.

Cette nouvelle tendance est également perceptible, par exemple, dans la seconde édition du Dictionnaire de la comptabilité, publié en 1982 sous le titre Dictionnaire de la comptabilité et des disciplines connexes. L'auteur principal de l'ouvrage affirme en préface qu'il souhaite "faire connaître ce qui est en usage ailleurs [qu'au Canada] de façon à ce que tous puissent saisir la portée des termes utilisés" (Sylvain, 1982: xvi). L'objectif de son ouvrage n'est pas d'uniformiser les terminologies de la comptabilité et des domaines connexes puisque, selon lui, "il convient que chaque pays conserve ce qui lui est propre, pourvu que la langue ne soit pas fautive" (ibid.).

\section{2- L'approche variationniste}

Des organismes de normalisation internationaux se sont prononcés en faveur de la reconnaissance de la variation géographique dans les travaux terminologiques unilingues français 
ou multilingues. Par exemple, l'ISO reconnaît que la variation géographique peut permettre de répondre plus adéquatement aux besoins terminologiques d'une communauté:

Les notions et les termes qui figurent dans les normes internationales ont été élaborés pour usage international, mais ils peuvent être inacceptables tels quels dans un contexte national. Ce principe s'applique également aux langues officielles de l'ISO aux différents niveaux nationaux (Organisation internationale de normalisation, 1996, iv).

Pour sa part, le Réseau panlatin de terminologie (Realiter) soutient l'approche variationniste qui consiste à refléter la variété des usages et des traditions terminologiques des différentes aires géographiques propres à chaque langue qui fait l'objet de travaux (Rousseau, 1995:s.p.). Selon les principes relatifs aux pratiques terminologiques adoptés par Realiter, le choix des termes à proposer doit tenir compte de l'usage observé, soit l'usage officialisé lorsqu'il existe, soit l'usage dominant parmi le public auquel le produit terminologique s'adresse (ibid.).

Comme les langagiers sont de plus en plus conduits à participer à des activités de traduction et de rédaction en tenant compte des spécificités culturelles de leurs publics cibles (ce qui correspond à un aspect important de ce qu'on appelle la localisation $^{5}$ ), on peut considérer que l'approche variationniste s'inscrit dans un mouvement général d'adaptation culturelle des produits et services.

En définitive, on peut faire un parallèle entre l'approche variationniste en contexte unilingue et celle de la valorisation et de la reconnaissance de la diversité linguistique en contexte multilingue. Dans les deux cas, on perçoit la volonté de préserver les identités culturelles nationales tout en encourageant la communication internationale. Dans cette optique, le Québec fait la promotion de la diversité linguistique pour veiller au maintien du statut du français sur son territoire et même pour

$5 \quad$ Voir à ce sujet Quirion (2003).

TradTerm, 14, 2008, p. 193-220 
favoriser le multilinguisme sur le plan supranational afin que le français occupe une place parmi les grandes langues de communication.

À l'heure actuelle, pour les auteurs de vocabulaires spécialisés, de lexiques et de banques de données terminologiques, le travail de description des variantes topolectales s'accompagne du défi constant de concevoir des outils qui répondent pleinement aux besoins du plus grand nombre de francophones possible, notamment en ce qui a trait à leur besoin d'intercommunication. Le succès de cette entreprise réside principalement dans la concertation et l'échange, au sein de la francophonie, entre les experts d'un domaine donné et les langagiers, dans un esprit de respect de l'évolution linguistique des différentes communautés.

\section{3- Les difficultés entourant la prise en compte de la variation topolectale}

Plusieurs ouvrages terminologiques produits ces dernières années s'inscrivent dans une tendance renforcée de valorisation de la diversité linguistique, laquelle se fonde sur une volonté de participation à l'enrichissement du français comme outil de développement national, supranational et international. Cette perspective relativement nouvelle entraîne avec elle un ajustement des pratiques méthodologiques sous-jacentes au travail terminologique. Car, si concevoir l'importance de tenir compte de la variation géographique est une chose, réussir à la cerner correctement en est une autre. Ainsi, le travail terminologique doit s'appuyer sur la documentation la plus vaste et la plus diversifiée possible et, au besoin, bénéficier du concours de spécialistes de divers domaines provenant de différents États de la francophonie. En effet, la variation terminologique sur le plan géographique constitue un phénomène linguistique complexe. Pour saisir de façon précise de quelle façon elle touche un champ terminologique donné, il faut généralement disposer de grands corpus représentatifs de l'usage des divers groupes professionnels concernés et des différentes communau- 
tés géopolitiques prises en considération. Ces types de corpus ne sont pas toujours accessibles aux terminologues, et ces derniers doivent souvent s'en remettre aux indications à caractère topolectal qu'ils trouvent dans les ouvrages terminologiques et lexicographiques déjà existants. Ils peuvent néanmoins chercher à cerner la variation terminologique topolectale à l'aide des moteurs de recherche dans Internet, tout en gardant à l'esprit qu'il faut exploiter les données géolinguistiques du Web avec discernement et beaucoup de prudence.

\section{4- Le marquage topolectal}

De façon générale, les terminologues qui relèvent des variantes topolectales dans les domaines d'activités sur lesquels portent leurs travaux ${ }^{6}$ recourent à des marques topolectales pour caractériser ces variantes. Toutefois, bien peu d'entre eux font explicitement état des principes qui guident leur façon de marquer géographiquement les termes. Dans les ouvrages portant sur la terminologie, le traitement de la variation topolectale ne semble pas non plus avoir fait l'objet de travaux de réflexion poussés. Généralement, on se contente de souligner l'utilité du marquage topolectal ${ }^{7}$ en indiquant uniquement qu'il est important d'associer à une variante topolectale un code alphabétique correspondant à la zone géographique où elle est employée (ce qu'il convient de nommer marquage topolectal de terme).

Certains principes généraux sont néanmoins connus et partagés par plusieurs organismes dédiés à la production terminologique. Ainsi, l'ISO préconise le recours à la catégorie usage géographique "pour indiquer des différences d'emploi des termes, à l'échelle locale, régionale ou nationale" et suggère que soient identifiés les usages géographiques à l'aide de symboles

6 La présence de variantes topolectales dépend de la situation linguistique du secteur de l'activité humaine sur lequel porte le travail terminologique.

7 Voir, par exemple, Organisation internationale de normalisation (1999a: 13), Dubuc (1985:104) et Rondeau (1984:86).

TradTerm, 14, 2008, p. 193-220 
de pays ou par un nom de continent (Organisation internationale de normalisation, 2001:15). S'il est disponible et pertinent, l'identifiant de l'usage géographique devrait prendre la forme d'un symbole de pays (ou de l'une de ses subdivisions) spécifié dans la norme ISO 3166 (Organisation internationale de normalisation 1997, 1998, 1999b). À ce sujet, l'ISO suggère des codes alphabétiques à deux ou à trois lettres. Ainsi, on peut référer à la Belgique par $B E$ ou $B E L$, au Canada par $C A$ ou $C A N$, et au Québec par $Q C$. Cette façon de faire a également été adoptée par la Conférence des services de traduction des États d'Europe occidentale (Conférence des services de traduction des États d'Europe occidentale. Groupe de travail terminologie et documentation, 1990:9).

Par ailleurs, Realiter se prononce en faveur d'un marquage topolectal souple dans lequel une marque n'a pas de valeur exclusive, le terme marqué pouvant circuler ou être utilisé dans d'autres zones de la latinité. À cet effet, Loïc Depecker ${ }^{8}$, lors de la $7^{\mathrm{e}}$ réunion de coordination de Realiter tenue à Rome en juin 1998, a précisé que le sens d'une marque topolectale indique de façon générale, et sauf indication contraire, que le terme est particulièrement utilisé (ou a fait l'objet d'une proposition) dans la zone géographique notifiée (Depecker, 1998). Pour l'essentiel, la même valeur est donnée à la marque topolectale dans la production terminologique de l'Office québécois de la langue française (OQLF).

\section{5- L'importance du phénomène de la variation géographique}

Malgré quelques différences, il ne faut pas perdre de vue que la grande majorité des termes et expressions diffusés, par exemple, dans Le grand dictionnaire terminologique (GDT), sont dans les faits en usage à la fois en France, au Québec et dans les autres pays de la francophonie ${ }^{9}$. L'harmonisation des termes demeure d'ailleurs un idéal toujours poursuivi par la pratique

\footnotetext{
8 Alors secrétaire général du Réseau panlatin de terminologie.

9 Voir à ce sujet Vézina (2004).
} 
terminologique. Un relevé très approximatif nous a permis d'établir quelques statistiques quant à la présence de la variation topolectale à l'intérieur de la partie française du Grand dictionnaire terminologique. À ce jour, nous estimons à près de 0,75 \% la proportion de fiches diffusées dans lesquelles au moins un indicatif de pays est utilisé pour rendre compte de la variation géographique touchant un terme français. Si on ajoute à ce pourcentage la proportion de fiches diffusées qui comportent des indications topolectales fournies à l'intérieur de notes, nous obtenons un total d'un peu moins de $2 \%$. De même, dans la banque Termium Plus, produite par le Bureau de la traduction du Canada, le pourcentage de fiches terminologiques qui comportent au moins une marque topolectale dans leur volet français est d'environ $1,1 \%{ }^{10}$. Ces faibles pourcentages indiquent que la variation topolectale ne touche qu'une très petite partie du lexique spécialisé francophone. Cependant, il ne faut pas oublier que le contenu d'une banque terminologique comme le GDT (qui compte environ 3 millions de termes français et anglais) témoigne de trois décennies de production terminologique et qu'une partie de ce contenu reflète les orientations normatives des années 70 et du début des années 80 , moins ouvertes à la variation. Si tout le contenu du GDT reflétait les orientations actuelles, le pourcentage de fiches terminologiques qui comporteraient une marque topolectale serait sans doute un peu plus élevé.

La validité de cette assertion se trouve en partie vérifiée lorsqu'on examine l'état de la variation topolectale dans la toute dernière édition du Dictionnaire de la comptabilité et de la gestion financière (2004), dont environ $7 \%$ des entrées françaises comportent une marque topolectale ${ }^{11}$. L'attention toute particulière qui a été portée au phénomène de la variation géographique dans ce domaine spécialisé explique pour une bonne part pourquoi ce pourcentage est plus élevé que ce qu'on trouve dans le GDT. L'écart s'explique également en raison du fait que le do-

\footnotetext{
10 D’après les renseignements qui nous ont été fournis par Gabriel Huard, directeur de la normalisation terminologique au Bureau de la traduction. 11 D'après les renseignements qui nous ont été fournis par l'auteur.
} 
maine de la comptabilité et de la gestion financière, qui comporte une dimension culturelle certaine, est plus sujet à la variation géographique que, par exemple, la chimie ou les mathématiques.

Somme toute, on peut avancer que la variation topolectale ne touche qu'une frange du lexique spécialisé francophone. Ce constat vient renforcer l'idée selon laquelle les différences topolectales sur le plan terminologique constituent des signes de la richesse lexicale du français qui sont peu susceptibles d'affecter l'intercompréhension entre les francophones de différentes communautés géopolitiques. La reconnaissance des variantes topolectales dans les travaux propres aux différentes variétés nationales de français apparaît d'autant plus légitime.

\section{6- Les cas de non-marquage}

La décision et la façon de recourir à des marques topolectales dans un travail terminologique dépendent principalement des objectifs poursuivis et du public visé. Ainsi, une fiche terminologique ou un glossaire élaboré par des francophones belges et pour des francophones belges pourrait proposer des marques uniquement pour les usages géographiques différents de ceux qui ont cours en Belgique, les usages belges étant alors considérés comme la référence à partir de laquelle on établit les différences. Dans un contexte comme celui-là, un terme qui ne serait employé que sur le territoire belge ne serait pas identifié par une marque topolectale.

En lexicographie, les auteurs du Dictionnaire québécois d'aujourd'hui (DQA) ont choisi de prendre comme objet de description l'intégralité du français en usage au Québec. En ne limitant pas leur travail au relevé des différences et en posant ainsi la variété québécoise au centre de leur description, ils ont opté pour l'usage d'un marquage topolectal inversé qui s'appliquait dès lors aux particularités du français en usage en France, variété de référence dans les ouvrages conçus en France. C'est ainsi que, dans le DQA, le terme traversier "navire spécialement aménagé pour faire traverser des passagers, des véhicules d'une 
rive à l'autre d'un cours d'eau" a été présenté sans marque, alors que son synonyme ferry-boat est porteur de la marque topolectale France (DQA, 1993:485 et 1202).

Dans le cadre d'un travail terminologique qui s'adresse à un public panfrancophone, l'absence de marquage peut prendre un tout autre sens. Ainsi, dans Le grand dictionnaire terminologique et la base de données Termium Plus, un terme sans marque topolectale est considéré comme appartenant à l'usage de l'ensemble des communautés géopolitiques où le français a le statut de langue nationale (Belgique, Canada, France, Québec, Suisse, etc.) ${ }^{12}$. Par exemple, les termes daltonisme et produit intérieur brut, dont l'usage est partagé par les locuteurs de toutes les zones francophones, ne portent pas de marques topolectales dans ces deux banques terminologiques.

De même, dans le cas d'un terme néologique, si on a pour objectif de le voir s'implanter dans l'ensemble de la francophonie, on s'abstient généralement de lui attribuer une marque topolectale indiquant la communauté où le terme a été créé; cette façon de faire repose également sur le constat selon lequel les néologismes se diffusent désormais rapidement d'une communauté à l'autre, notamment grâce à Internet (par exemple, dans le GDT, le terme hameçonnage ${ }^{13}$, créé en avril 2004 par une terminologue de l'Office, madame Yolande Perron, est déjà attesté en Europe). D'ailleurs, on ne saurait trop insister sur l'impossibilité d'établir une équivalence stricte entre l'origine géographique d'un terme et son aire d'utilisation, et ce, que ce soit en contexte de néologie ou non. Ainsi, le terme courriel, d'abord implanté au Québec, est désormais en usage non seule-

12 On entendra par langue nationale la langue officielle d'un État qui constitue également la langue maternelle de la majorité de sa population et qui est largement utilisée dans le système d'éducation et dans les activités socioéconomiques et culturelles de cet État.

13 Hameçonnage : Envoi massif d'un faux courriel, apparemment authentique, utilisant l'identité d'une institution financière ou d'un site commercial connu, dans lequel on demande aux destinataires de mettre à jour leurs coordonnées bancaires ou personnelles, que le pirate utilisera pour détourner des fonds à son avantage.

TradTerm, 14, 2008, p. 193-220 
ment au Canada, mais également en Europe. De même, le terme baladeur (équivalent français de Walkman), créé en France et officialisé en 1983, est implanté au Québec depuis déjà plusieurs années.

\section{II- Les types de marquage topolectal}

Lorsque la variation terminologique touche clairement un concept donné sur le plan géographique, une indication d'ordre géographique peut être intégrée sur la fiche de terminologie par le marquage topolectal de terme ou par le marquage topolectal conceptuel.

Le marquage topolectal de terme consiste à associer à l'usage d'un terme un certain territoire ou une certaine communauté géopolitique. Ce type de marquage vise donc à décrire l'extension géographique d'un terme, d'une variante graphique, etc. Quant au marquage topolectal conceptuel, il consiste à associer au concept traité une " appartenance " géographique. L'utilisation de ces deux types de marquages est très répandue en lexicographie ${ }^{14}$. Par exemple, les dictionnaires des maisons Robert et Larousse les ont adoptés depuis plusieurs années déjà ${ }^{15}$. Cette pratique est également utilisée dans des ouvrages terminologiques (le Dictionnaire de la comptabilité et de la gestion financière de Louis Ménard, par exemple) ou dans certaines banques terminologiques, comme Eurodicautom ${ }^{16}$.

\footnotetext{
14 Voir Rézeau (1986:44), qui indique que dans les cas où des mots désignent des "realia propres à une région [...], l'étiquette régionale n'est guère adéquate: le caractère régional fait partie de la dénotation et il devrait entrer dans la définition".

15 Voir par exemple les articles cégep (marquage conceptuel) et fève (marquage de terme) dans Le petit Robert, 1977.

16 Le marquage topolectal conceptuel n'y est toutefois pas uniforme.
} 


\section{1- Le marquage topolectal de terme}

De façon générale, pour un concept donné, dans une situation où il existe plusieurs termes distribués sur des territoires différents, chaque terme reçoit une marque topolectale correspondant à chacune des communautés où il est en usage. Par exemple, dans Termium Plus, sous ombudsman, le terme synonyme protecteur du citoyen est identifié comme propre à l'usage canadien, Commissaire du Parlement, comme propre au français de Belgique et médiateur, comme propre au français en usage en France.

De la même manière, le Dictionnaire de la comptabilité et de la gestion financière de Louis Ménard (2004) recourt aux marques géographiques abrégées (codes alphabétiques à deux ou trois lettres) pour indiquer qu'un usage est propre à un pays ou à un État. Une liste en début d'ouvrage expose les différentes marques géographiques utilisées. Par exemple, dans l'article bond discount sont présentés l'équivalent français en usage au Canada, escompte d'émission d'obligations, et l'équivalent français en usage en France et en Belgique, prime d'émission.

Quant aux variantes topolectales présentées dans Le grand dictionnaire terminologique, l'information qui permet de situer géographiquement des usages concomitants est fournie en abrégé au moyen d'un code alphabétique; l'usage des termes marqués géographiquement peut aussi faire l'objet de notes explicatives. Les indicatifs qui sont actuellement le plus couramment utilisés sont QC (Québec), CA (Canada), FR (France), BE (Belgique) et $\mathrm{CH}$ (Suisse). Au besoin, d'autres indicatifs sont disponibles, comme ceux présentés dans la norme ISO 3166. On peut également avoir recours à des notes explicatives pour préciser la valeur du marquage topolectal utilisé. Par exemple, le terme commanditaire, en usage au Québec, est accompagné de l'indicatif géographique $Q C$, alors que le terme parraineur, en usage en France, est suivi de FR et d'une note explicative qui mentionne que le terme a fait l'objet d'une recommandation officielle en France.

Ce type de marquage, souvent le seul qui est mentionné dans les manuels de terminologie, n'est pas satisfaisant pour tous les cas de variation géographique. Ainsi, il ne permet pas 
de rendre compte du caractère particulier d'un concept qui correspond à une réalité propre à une communauté donnée; dans un tel cas, c'est le concept lui-même qui est associé directement à un territoire. La plupart du temps, le terme qui sert à le désigner devient donc le seul auquel les usagers d'une même langue peuvent recourir pour référer au concept en cause, et ce, indépendamment de la variété de langue qu'ils utilisent. On doit alors plutôt recourir au marquage topolectal conceptuel.

\section{2- Le marquage topolectal conceptuel}

Certains secteurs d'activités (assurances, comptabilité, droit, etc.) sont organisés de façon différente d'un État à l'autre, ce qui entraîne de la variation terminologique ${ }^{17}$. Ainsi, le marquage topolectal conceptuel vise à décrire l'extension géographique d'un concept associé à des réalités politiques, administratives, socioéconomiques, matérielles et culturelles qui sont propres à un État, à un ensemble d'États, à un peuple ou à un territoire donné. Ce type de marquage se rencontre notamment à l'intérieur de définitions et dans des notes explicatives. Mentionnons également la possibilité de recourir à un type de marquage qui s'apparente à une indication de domaine d'emploi, par lequel il est possible d'indiquer l'extension géographique d'un concept en choisissant, dans un menu déroulant, le nom de l'État ou d'un ensemble d'États où le concept traité existe.

\section{1- Le marquage intradéfinitionnel}

La marque topolectale intradéfinitionnelle peut être placée au tout début de la définition, en tant que proposition circonstancielle de lieu ${ }^{18}$, ou bien à l'intérieur du texte de la définition. Ce type de marquage est tout indiqué lorsque le concept renvoie nettement à une réalité propre à une communauté géo-

\footnotetext{
17 Voir Cabré (1998:225, note 14).

18 En terminologie, la présence d'une telle marque au début de l'énoncé définitoire n'est toutefois pas toujours souhaitable, étant donné qu'elle empêche l'incluant de figurer en tête de définition.
} 
politique. Cette appartenance à une communauté particulière ou à un territoire donné est alors vue comme un trait définitoire. Selon ce principe, dans Le grand dictionnaire terminologique, on définit le terme whip (domaine: politique) ainsi: "Au Canada et dans les pays de tradition parlementaire britannique, député désigné par le chef de chaque parti pour assurer la cohésion du groupe ainsi que la discipline et l'assiduité de ses membres". De même, la définition du terme attestation d'équivalence (domaine : éducation) va comme suit: "Pièce officielle établissant la correspondance ou l'égalité de valeur entre les études faites par une personne à l'intérieur ou à l'extérieur de l'actuel système scolaire du Québec et une classe ou un programme d'études de ce système".

Certains auteurs préfèrent avoir recours à la marque topolectale de type marque de terme, c'est-à-dire aux noms de pays complets ou aux codes alphabétiques qui leur correspondent, pour indiquer les concepts qui s'appliquent à des territoires géographiques déterminés. Les marques topolectales utilisées dans des cas comme ceux-là peuvent être placées devant la définition, de manière à signaler que c'est le concept qui est marqué géographiquement. C'est cette méthode qui est utilisée dans le Dictionnaire de la comptabilité et de la gestion financière (2004). L'article goods and services tax est un bon exemple de ce type de marquage, où la marque (CA) est présentée devant la définition, plutôt qu'à côté du terme principal.

On peut par ailleurs mentionner un certain type d'indication intradéfinitionnelle qui s'apparente à une marque topolectale conceptuelle sans en être strictement une. Cette indication, qui ne sert pas à qualifier une variante lexicale géographique, permet plutôt de préciser l'extension géographique de l'objet rattaché à un concept. Cette situation se présente lorsqu'on définit un concept qui réfère à une réalité naturelle rattachée à un territoire donné. Lorsque la réalité à décrire connaît une distribution géographique particulière, la mention de cette aire de distribution constitue un trait définitoire important. Ce territoire (un continent ou une partie de continent, un hémisphère, un océan, etc.) ne correspond généralement pas à un territoire national d'une communauté linguistique et il est le plus souvent plus vaste que ce dernier. Ainsi, dans Le grand dictionnaire 
terminologique, le terme bleuet est accompagné de la définition suivante : "Baie comestible bleu-noir d'Amérique du Nord [...]".

\section{2- La présence d'indications de nature topolectale dans les notes explicatives}

Des indications de nature topolectale peuvent être données à l'intérieur de notes explicatives à caractère conceptuel lorsque, pour un certain concept décrit, de légères différences sont perceptibles dans les usages de différentes communautés géopolitiques. La note sert alors à renseigner l'usager sur les particularités fines ou complexes du concept selon le territoire où sont en usage les termes (ou le terme) qui le désignent. Dans Termium Plus, une note suit la définition donnée au terme passif: "En France et en Belgique, le passif comprend non seulement les capitaux empruntés [...] mais aussi les capitaux propres".

\section{III- La problématique de la variation topolectale en contexte de terminologie multilingue}

Tous les travaux terminologiques, qu'ils visent la rédaction d'une fiche de terminologie, l'élaboration d'ouvrages terminologiques ou le développement du contenu de banques de données terminologiques, devraient être menés en suivant une politique éditoriale qui en détermine le cadre des valeurs et en définit les options terminologiques. De fait, les auteurs de produits terminologiques ont tout intérêt à se doter d'une telle politique éditoriale parce qu'elle explicite leur position concernant la norme linguistique et terminologique. Et c'est cette position qui influence les choix qu'ils doivent faire quant au terme principal à privilégier, à l'ordre de présentation des synonymes, aux critères d'acceptation des emprunts, à l'utilisation des marques topolectales, etc. Ces considérations valent autant pour les travaux unilingues que pour les travaux terminologiques bilingues ou multilingues.

Ces considérations prennent toute leur importance lorsqu'il est question de travaux terminologiques menés en collaboration avec plusieurs partenaires, que ce soit dans une 
optique unilingue ou multilingue. La terminologie telle qu'elle est pratiquée par exemple au sein de Realiter nous confronte à ces questions sur lesquelles les partenaires d'un projet doivent s'entendre. Il ne sera pas question ici de la recherche terminologique en tant que telle, mais de la présentation des résultats de cette recherche, même si une politique éditoriale couvre les deux volets. Selon nous, il est évident que la présentation des résultats est un aspect à ne pas négliger, étant donné que la clarté du message diffusé en dépend.

\section{1- La présentation d'ouvrages terminologiques multilingues: choix de la langue}

Le choix de la langue à partir de laquelle seront présentés les articles terminologiques constitue une des préoccupations des auteurs de produits terminologiques, parce qu'il oriente leur travail.

Lorsqu'au Québec le besoin d'une terminologie en français se fait sentir dans des domaines ou des secteurs fortement anglicisés ou occupés par une autre langue, l'OQLF choisit généralement de présenter le résultat des recherches à partir de l'anglais ou d'une autre langue (langue source) vers le français (langue cible). Par exemple, dans le Lexique de la restauration chinoise, publié en 1984, les données sont présentées de l'anglais vers le français, en passant par le chinois.

Par contre, dans des situations où le vocabulaire fait défaut en français, ou lorsque règne la confusion sémantique ou lexicale, l'Office publie des ouvrages terminologiques élaborés à partir du français et à l'intérieur desquels parait également l'anglais afin de répondre aux besoins de traduction qui, au Québec, vont souvent de l'anglais vers le français. Par exemple, le Vocabulaire de la chaussure (1982), le Vocabulaire de l'habillement (1994) et le Dictionnaire de l'industrie automobile (2001) présentent les données en partant du français.

Dans le cas de travaux multilingues comme le Lexique panlatin d'Internet, publié en 1999, il a été convenu que l'ouvrage se présenterait comme une série de fascicules lexicaux qui se succéderaient selon l'ordre alphabétique des huit langues (selon 
leur appellation en langue originale) dans lesquelles la recherche avait été menée. On trouve donc, dans l'ordre, les lexiques catalan (catalán), anglais (english), espagnol (español), français, galicien (galego), italien (italiano), portugais (português) et roumain (românî).

Étant donné les enjeux qui motivent les travaux de Realiter et qui peuvent être différents d'un État à un autre, il convient évidemment qu'on se prononce sur le type de présentation souhaitée.

\section{2- L'article terminologique: choix du terme principal dans une langue et ordre d'apparition des équivalents dans d'autres langues}

Pour chacune des langues en cause, le choix du terme principal obéit aux mêmes règles que dans le cadre de travaux terminologiques unilingues. C'est la politique éditoriale adoptée par les auteurs qui guide le choix du terme à privilégier. Dans une perspective de travaux multilingues, quel terme principal doit-on retenir pour chacune des langues et quelle est l'influence de la variation topolectale sur ce choix?

Chaque produit terminologique obéit à des politiques d'édition qui lui sont propres, politiques qui comprennent notamment des règles de marquage. Cela explique les différences que l'on peut observer entre les articles terminologiques relatifs à un même concept, selon qu'ils sont présentés dans un produit terminologique ou dans un autre. Si on compare le traitement du concept de "conversation entre internautes " dans quatre produits terminologiques différents, on peut constater qu'il existe des différences dans le choix du terme principal ainsi que dans le marquage ou le non-marquage du terme. 


\begin{tabular}{|l|l|}
\hline Produit terminologique & Terme principal \\
\hline $\begin{array}{l}\text { Le grand dictionnaire terminologique } \\
\text { (fiche de 1998) } \\
\text { (fiche de 2003) }\end{array}$ & bavardage-clavier $\mathrm{n} . \mathrm{m}$. \\
\hline CRITER (1999) & clavardage $\mathrm{n} . \mathrm{m}$. \\
\hline Lexique panlatin d'Internet (1999) & bavardage-clavier $\mathrm{n} . \mathrm{m}$. (QC) \\
\hline Termium Plus (2004) & bavardage $\mathrm{n} . \mathrm{m}$. \\
\hline
\end{tabular}

À partir des données de ce tableau, on constate que le terme principal et les critères de marquage topolectal ne sont pas les mêmes dans tous les ouvrages, notamment lorsqu'il s'agit de propositions néologiques; les choix effectués dépendent avant tout de la politique éditoriale adoptée.

De même, à l'intérieur d'un article terminologique, lorsque des équivalents de plusieurs langues sont présentés, il convient que soit déterminé l'ordre de leur présentation. C'est l'ordre alphabétique des noms de langues (en langue originale) qu'ont adopté les auteurs du Lexique panlatin du commerce électronique disponible gratuitement dans Internet. Ces derniers ont toutefois choisi de placer les termes de la langue anglaise à part, pour faire la distinction entre les langues latines et l'anglais.

\begin{tabular}{|c|c|c|}
\hline \multicolumn{3}{|c|}{$\begin{array}{l}\text { Lexique panlatin du commerce } \\
\text { électronique (2004) } \\
\text { Section " langue française " }\end{array}$} \\
\hline \multicolumn{3}{|l|}{ Terme principal fra } \\
\hline Équivalents & $\begin{array}{l}\text { catalán } \\
\text { español } \\
\text { galego } \\
\text { italiano } \\
\text { português } \\
\text { română }\end{array}$ & $\begin{array}{l}\text { cat } \\
\text { spa } \\
\text { glg } \\
\text { ita } \\
\text { por } \\
\text { ron }\end{array}$ \\
\hline & English & eng \\
\hline
\end{tabular}

TradTerm, 14, 2008, p. 193-220 


\section{3- La présentation des synonymes par langue et présentation des marques topolectales}

En ce qui concerne la présentation des autres termes que le terme principal, par exemple les synonymes, même si aucune règle écrite n'explique leur ordre de présentation, nous savons que plusieurs facteurs l'influencent néanmoins. Parmi ces facteurs, notons la fréquence d'usage du terme, sa vitalité, l'aire géographique de son emploi, etc. Actuellement, à l'OQLF, ce sujet est examiné dans le cadre des réflexions suscitées par le développement du nouveau module de production du Grand dictionnaire terminologique multilingue.

En observant deux articles terminologiques, l'un extrait du Lexique panlatin d'Internet (1999) et l'autre du Grand dictionnaire terminologique (2004), on constate que les synonymes d'une même langue sont généralement présentés selon un ordre déterminé par des considérations de nature linguistique ou extralinguistique propres à l'auteur de la recherche terminologique. En terminologie, cette façon d'établir l'ordre de présentation des synonymes est répandue.

\begin{tabular}{|c|c|}
\hline $\begin{array}{l}\text { Lexique panlatin d'Internet } \\
\text { (1999) }\end{array}$ & $\begin{array}{l}\text { Le grand dictionnaire terminologique } \\
\text { (2004) }\end{array}$ \\
\hline bavardage-clavier (37) $n . m .(Q C)$ & clavardage n. m. \\
\hline fra dialogue en direct $n . m$. & bavardage-clavier $n . m$. \\
fra causette $n . f .[F R A]$ & cyberbavardage $n . m$. \\
fra clavardage $n . m .(Q C)$ & bavardage en ligne $n . m$. \\
fra cyberbavardage $n . m$. & bavardage $n . m$. \\
fra bavardage $n . m$. & causette* $n . f$. \\
fra causerie bavardage $n . f$. & *mention, en note, de l'adoption \\
& officielle en France \\
\hline
\end{tabular}

Pour alléger les difficultés inhérentes à des projets terminologiques portant sur une même langue, notamment dans le cadre de projets internationaux menés en partenariat, il peut être souhaitable, comme certains l'ont d'ailleurs proposé, que l'ordre alphabétique soit privilégié dans la présentation des synonymes, indépendamment de la marque topolectale qui leur 
est associée ${ }^{19}$. Opter pour l'ordre alphabétique dans la présentation des synonymes d'une même langue fait en sorte, entre autres, d'éliminer la prédominance d'une variante topolectale par rapport à une autre, ce qui peut éventuellement être un effet recherché.

\begin{tabular}{|l|l|}
\hline \multicolumn{2}{|l|}{ Présentation possible des synonymes dans l'ordre alphabétique } \\
\hline bavardage-clavier $n . m .(Q C)$ & clavardage n. m. \\
\hline fra bavardage $n . m$. & fra bavardage $n . m$. \\
fra causerie bavardage $n . f$. & fra bavardage-clavier $n . m$. \\
fra causette $n . f .(F R A)$ & fra bavardage en ligne $n . m$. \\
fra clavardage $n . m .(Q C)$ & fra causette $n . f$. \\
fra cyberbavardage $n . m$. & fra cyberbavardage $n . m$. \\
fra dialogue en direct $n . m$. & \\
\hline
\end{tabular}

Lorsque plusieurs marques topolectales sont associées à un même terme, il serait souhaitable également qu'elles soient présentées l'une à la suite de l'autre, dans l'ordre alphabétique ${ }^{20}$. Ainsi, un terme propre à l'usage des communautés francophones de Belgique et de France serait suivi des marques BE et FR.

\begin{tabular}{|r|l|}
\hline Terme principal anglais : bond discount \\
\hline Équivalent français & prime d'émission (BE et FR) \\
\hline
\end{tabular}

Ce principe a l'avantage d'être facilement applicable dans le traitement terminologique des marques géographiques dans toutes les langues et il a d'ailleurs déjà été adopté dans le cadre de travaux terminologiques de Realiter.

19 Proposition faite en janvier 2003 par Louis-Jean Rousseau, alors coordonnateur linguistique du français d'Amérique et responsable du dossier des principes méthodologiques du travail terminologique (Realiter).

Ibid. 
Ces considérations nous amènent à nous demander si cette façon de présenter des données terminologiques et les marques géographiques qui les accompagnent convient au public cible auquel on destine les produits terminologiques, qu'ils soient unilingues, bilingues ou multilingues. Les usagers d'un produit terminologique doivent pouvoir saisir facilement le contexte de communication visé. Voilà les préoccupations qui doivent être au cour des discussions relatives à la mise au point d'une politique éditoriale sous-jacente à tout projet terminologique mené en partenariat.

\section{IV- Conclusion}

La recherche d'équilibre entre la valorisation des variétés nationales et le renforcement de l'appartenance à la francophonie justifie le recours au marquage topolectal dans les travaux de nature terminologique, et ce, même si la variation topolectale touche en fait très peu de termes et de concepts dans le registre spécialisé de la langue. On ne peut donc qu'encourager la poursuite de la réflexion sur les marques topolectales et sur leur présentation. Il faut en effet toujours viser à faciliter l'interprétation adéquate des données figurant dans un produit terminologique; le marquage topolectal s'avère d'ailleurs un outil utile à l'atteinte de cet objectif.

Dans les produits terminologiques de langue française, rappelons que le marquage topolectal doit rester souple, d'autant plus qu'il est souvent difficile de disposer d'un corpus suffisamment vaste, diversifié et détaillé pour pouvoir rendre compte avec précision de la répartition géographique d'une variante. Par ailleurs, si une variante est utilisée dans plus d'une aire géographique, elle devrait être accompagnée de toutes les marques topolectales pertinentes, et ces dernières devraient être présentées dans l'ordre alphabétique. Toutefois, lorsqu'un terme est en usage dans toutes les aires géographiques, évidemment, aucune marque n'est nécessaire. De plus, il convient que les marques topolectales ne soient jamais considérées comme définitives, étant donné l'évolution constante de la langue et des usages. L'abolition progressive des distances et des frontières, rendue possible 
par les nouvelles technologies de l'information (dont Internet est un élément majeur) et par la multiplication des partenariats internationaux, crée des conditions idéales pour la diffusion des terminologies et favorise les contacts entres les différentes variétés d'une langue ainsi qu'entre des langues différentes.

Dans un produit terminologique multilingue développé en partenariat, nous sommes d'avis que les équivalents de différentes langues devraient être présentés selon l'ordre alphabétique des noms de langues. De même, chaque nom de langue présenté devrait correspondre à celui en usage dans cette langue. Bien entendu, certains aspects que nous n'avons fait qu'effleurer doivent faire l'objet de réflexions supplémentaires par l'ensemble des intervenants afin qu'on puisse dégager un consensus quant au traitement de la variation topolectale dans les produits terminologiques multilingues.

Enfin, étant donné que la langue n'est pas uniquement un instrument de communication, mais qu'elle constitue également un moyen d'expression de la culture des communautés, nous croyons que le marquage topolectal est un moyen de souligner positivement la richesse lexicale d'une langue telle qu'elle se manifeste dans ses différentes variétés. À l'heure où on insiste de plus en plus sur l'importance de l'adaptation culturelle, et donc linguistique, des produits et des services, les avantages du marquage topolectal deviennent indéniables.

\section{Remerciements}

Les auteurs de ce texte ont bénéficié des commentaires de madame Tina Célestin, directrice des travaux terminologiques à l'Office québécois de la langue française, qu'ils tiennent à remercier. Tina Célestin et Annie Galarneau ont par ailleurs présenté le contenu de cet article à la Journée du Réseau panlatin de terminologie (Realiter) qui s'est tenue à Barcelone le 24 novembre 2004. 


\section{Références}

CABRÉ, Maria Teresa (1998) La terminologie: théorie, méthode et applications, Ottawa et Paris, Les Presses de l'Université d'Ottawa et Armand Colin, 322 p.

CONFÉRENCE DES SERVICES DE TRADUCTION DES ÉTATS D'EUROPE OCCIDENTALE. GROUPE DE TRAVAIL TERMINOLOGIE ET DOCUMENTATION (1990) CST : recommandations relatives à la terminologie, [Suisse], Conférence des services de traduction des États de l'Europe occidentale, Groupe de travail terminologie et documentation, 1 volume (f. mobiles).

DEPECKER, Loïc (1998) [Sans titre], texte proposé par Loïc Depecker et adopté par les membres du Réseau présents à la $7^{\mathrm{e}}$ réunion de coordination de Realiter (Rome, 26-27 juin 1998). Ce texte constitue maintenant la section II (Traitement des anglicismes) d'un document intitulé Principes méthodologiques du travail terminologique, consultable en ligne sur le site de Realiter [http://www.realiter.net/ $\mathrm{fr} /$ principios.htm].

DQA (1993) Dictionnaire québécois d'aujourd'hui, rédaction dirigée par Jean-Claude Boulanger, supervisée par Alain Rey, Saint-Laurent (Québec), Dicorobert inc., nouv. éd., xxxvii-1273 p. Complété par un Atlas géographique \& historique, une Chronologie, un Dictionnaire de noms propres et des Annexes grammaticales.

DUBUC, Robert (1985) Manuel pratique de terminologie, s. 1., Linguatech, $158 \mathrm{p}$.

GALARNEAU, Annie (2000) Traitement des nord-américanismes et prise en compte du contexte nord-américain dans le Nouveau Petit Robert, mémoire de maîtrise, Sainte-Foy, Université Laval, vii-136 p.

HAUSMANN, Franz Josef (1986) "Les dictionnaires de français hors de France”, La lexicographie québécoise: bilan et perspectives: actes du colloque organisé par l'équipe du Trésor de la langue française au Québec et tenu à l'Université Laval les 11 et 12 avril 1985, Lionel Boisvert, Claude Poirier et Claude Verreault (dir.), Québec, Les Presses de 1'Université Laval, p. 3-21 (Langue française au Québec. $3^{\mathrm{e}}$ section, Lexicologie et lexicographie; 8). 
Le grand dictionnaire terminologique, [En ligne], Québec, Office québécois de la langue française [http://www.oqlf.gouv.qc.ca/ ressources/gdt_bdl2.html] (Consulté le 20 octobre 2004).

Lexique panlatin d'Internet, [En ligne], [Gatineau, Québec], Travaux publics et Services gouvernementaux Canada, Bureau de la traduction [http://www.bureaudelatraduction.gc.ca/ pwgsc_internet/fr/publications/gratuit_free/lex_panlatin_f.htm] (Consulté le 25 juillet 2006).

MÉNARD, Louis (2004) Dictionnaire de la comptabilité et de la gestion financière : anglais-français avec index français-anglais, avec la collab. de Murielle Arsenault, Jean-François Joly et Jean-Jacques Lavoie; publ. en collab. avec l'Ordre des experts comptables (France), la Compagnie nationale des commissaires aux comptes (France) et l'Institut des reviseurs d'entreprises (Belgique), $2^{\text {e }}$ éd., Montréal et Toronto, Institut canadien des comptables agréés, xxvi-1581 p.

OFFICE DE LA LANGUE FRANÇAISE (1965) Norme du français écrit et parlé au Québec, [Québec], Ministère des Affaires culturelles du Québec, 12 p.

OFFICE DE LA LANGUE FRANÇAISE (1982) Vocabulaire de la chaussure: français-anglais, [Montréal], OLF, 47 p.

OFFICE DE LA LANGUE FRANÇAISE (1984) Vocabulaire de l'habillement : français-anglais, $2^{\mathrm{e}}$ éd. Revue et corrigée, Québec, Direction générale des publications gouvernementales, $173+[2] \mathrm{p}$.

OFFICE DE LA LANGUE FRANÇAISE (2001) Dictionnaire de l'industrie automobile: terminologie du transport: français-anglais, Sainte-Foy, Publications du Québec, 538 p.

ORGANISATION INTERNATIONALE DE NORMALISATION (1996) Travaux terminologiques - Harmonisation des notions et des termes $=$ Terminology work - Harmonization of concepts and terms, [Genève], ISO, iv-7 p. (Recommandation ISO/860).

ORGANISATION INTERNATIONALE DE NORMALISATION (1997) Codes for the representation of names of countries and their subdivisions Part 1: Country codes $=$ Codes pour la représentation des noms de pays et de leurs subdivisions - Partie 1: Codes pays, [Genève], ISO, ix-58 p. ( Norme internationale ISO 3166-1).

ORGANISATION INTERNATIONALE DE NORMALISATION (1998) Codes for the representation of names of countries and their subdivisions Part 2: Country subdivision code $=$ Codes pour la représentation des 
noms de pays et de leurs subdivisions - Partie 2: Code pour les subdivisions de pays, [Genève], ISO, ix-105 p. (Norme internationale ISO 3166-2).

ORGANISATION INTERNATIONALE DE NORMALISATION (1999a) Aides informatiques en terminologie - Catégories de données $=$ Computer applications in terminology - Data categories, [Genève], ISO, iv-58 p. (Norme internationale ISO 12620).

ORGANISATION INTERNATIONALE DE NORMALISATION (1999b) Codes for the representation of names of countries and their subdivisions Part 3: Code for formerly used names of countries = Codes pour la représentation des noms de pays et de leurs subdivisions - Partie 3: Code pour les noms de pays antérieurement utilisés, [Genève], ISO, ix-11 p. (Norme internationale ISO 3166-3).

ORGANISATION INTERNATIONALE DE NORMALISATION (2001) Terminographie axée sur la traduction = Translation-oriented terminography, [Genève], ISO, 20 p. (ISO/FDIS 12616. ISO/TC 37/ SC 2. Projet final).

QUIRION, Jean (2003) La formation en localisation à l'université : pour quoi faire?, Meta, volume 48, $\mathrm{n}^{\circ} 4$, Montréal, Les Presses de l'Université de Montréal, p. 546-558.

RÉZEAU, Pierre (1986) Les régionalismes et les dictionnaires du français ", La lexicographie québécoise: bilan et perspectives. Actes du colloque organisé par l'équipe du Trésor de la langue française au Québec et tenu à l'Université Laval les 11 et 12 avril 1985, Lionel Boisvert, Claude Poirier et Claude Verreault (dir.), Québec, Les Presses de 1'Université Laval, p. 41-52 (Langue française au Québec. $3^{\mathrm{e}}$ section, Lexicologie et lexicographie; 8).

ROBERT, Paul (1977) Le Petit Robert. Dictionnaire alphabétique et analogique de la langue française, Paris: Le Robert, [xxxi]-2173 p. [rédaction dirigée par Alain Rey et Josette Rey-Debove].

RONDEAU, Guy (1984) Introduction à la terminologie, Chicoutimi (Québec): Gaëtan Morin éditeur, xlvi-238 p.

ROUSSEAU, Louis-Jean (1995) [Sans titre], texte proposé par LouisJean Rousseau et adopté par les membres du Réseau présents à la $4^{\text {e }}$ réunion de coordination de Realiter (Barcelone, 14-16 décembre 1995). Ce texte constitue maintenant la section I (Principes généraux) d'un document intitulé Principes méthodologiques du travail 
terminologique, consultable en ligne sur le site de Realiter [http:// www.realiter.net/fr/principios.htm].

SYLVAIN, Fernand (1977) Dictionnaire de la comptabilité, avec la collab. de Murielle Arsenault et Marie-Éva de Villers-Sidani, Toronto, Institut canadien des comptables agréés, $258 \mathrm{p}$.

SYLVAIN, Fernand (1982) Dictionnaire de la comptabilité et des disciplines connexes, avec la collaboration de Murielle Arsenault, JeanFrançois Joly, Dominique Ledouble et Henri Olivier, $2^{\mathrm{e}}$ éd. ent. rev., corr. et augm., Toronto, Institut canadien des comptables agréés; Paris, Ordre des experts comptables et des comptables agréés; Bruxelles, Institut des reviseurs d'entreprises, xxi-662 p.

Termium Plus, [En ligne], [Gatineau, Québec], Travaux publics et Services gouvernementaux Canada, Bureau de la traduction [http:// www.termium.gc.ca/site/accueil_home_f.html] (Consulté le 20 octobre 2004).

VÉZINA, Robert (2004) La prise en compte de la variation lexicale dans la production terminologique et linguistique de l'Office de la langue française. La variation dans la langue standard. Actes du colloque tenu les 13 et 14 mai 2002 à l'Université Laval dans le cadre du $70^{e}$ Congrès de l'ACFAS, sous la direction de Pierre Bouchard et Robert Vézina, Montréal, Office québécois de la langue française, p. 181193.

VÉZINA, Robert (2005) L'OQLF et les particularismes du français du Québec: aspects rétrospectifs. La lexicographie différentielle du français et le Dictionnaire des régionalismes de France, Colloque en l'honneur de Pierre Rézeau pour son soixante-cinquième anniversaire, sous la direction de Martin-D. Glessgen et André Thibault, Strasbourg, Presses Universitaires de Strasbourg, p. 267-280. 